\title{
Cirugía coronaria. Evolución en la última década. Indicaciones y resultados actuales
}

\author{
Jesús Herreros \\ Servicio de Cirugía Cardiovascular. Clínica Universitaria. Universidad de Navarra. Pamplona. España.
}

\begin{abstract}
La cirugía coronaria está avalada por unos excelentes resultados, bien documentados a largo plazo como consecuencia de la revascularización completa y la utilización de una o 2 arterias mamarias. En este artículo se revisan los resultados y las indicaciones de la cirugía y se valoran los nuevos retos y las oportunidades que incluyen la cirugía más segura, menos agresiva y asociada a otros procedimientos quirúrgicos. El objetivo es desarrollar estrategias ligadas a un ciclo innovador que adapte la cirugía a las necesidades de la población, las nuevas tecnologías y a las actividades pioneras.
\end{abstract}

Palabras clave: Cirugía coronaria. Cirugía coronaria sin bomba. Mamaria interna. Insuficiencia mitral isquémica. Anuloplastia mitral restrictiva. Reconstrucción ventricular.

\section{Coronary Surgery. Developments in the Last Decade. Indications and Results}

Coronary artery bypass surgery is endorsed by the excellent, well-documented, long-term results that follow complete revascularization and the use of 1 or 2 mammary artery grafts. This article contains a review of the current indications for and the results of such surgery and an evaluation of new challenges and opportunities, including the implementation of safer and less aggressive surgery, and surgery associated with other operative procedures. The aim was to develop a strategy linked to a cycle of innovation that could be used to adapt surgery to the needs of the population, to new technologies, and to pioneering developments.

Key words: Coronary artery bypass surgery. Off-pump coronary artery bypass surgery. Internal mammary artery. Ischemic mitral regurgitation. Mitral restrictive annuloplasty. Surgical ventricular reconstruction.

Full English text available at: www.revespcardiol.org

\section{INTRODUCCIÓN}

La cirugía coronaria tiene un origen incierto, en función de la localización geográfica y las preferencias del autor: Senning en Europa (1966), Garrett (1996) y Favaloro (1997) en Estados Unidos, o incluso mucho antes si consideramos los trabajos experimentales de Alexis Carrel. A los intentos realizados a partir de 1951 por Vineberg en Montreal, introduciendo la arteria mamaria interna (AMI) dentro del

\section{Sección patrocinada por el Laboratorio Dr. Esteve}

Correspondencia: Dr. J. Herreros.

Servicio de Cirugía Cardiovascular. Clínica Universitaria Navarra.

Av. Pío XII, 36. 31008 Pamplona. España.

Correo electrónico: jherreros@unav.es miocardio, siguieron otras propuestas de revascularización directa mediante endarterectomía, interposición de vena safena o AMI, sin que estas técnicas fueran aceptadas ni su aplicación potencial valora$\mathrm{da}^{1}$. En 1964, Kolesov en Leningrado, anastomosó la AMI a la arteria descendente anterior (DA) ${ }^{2}$. Sin conocimiento de esta contribución, Garrett, del equipo de DeBakey en Houston, utilizó la vena safena para revascularizar la DA en 1966, con permeabilidad del injerto a los 7 años $^{3}$ y Favaloro introdujo en la Cleveland Clinic el bypass aortocoronario en $1967^{4}$, con una experiencia en 1971 de 741 casos $^{5}$. En 1968, en Nueva York, Green anastomosó la AMI a la DA ${ }^{6}$; en 1971, Flemma, Johnson y Lepley en Milwaukee describieron las ventajas de los injertos secuenciales ${ }^{7}$; se tiene constancia del uso de las 2 AMI en 1972, aunque probablemente se había utilizado ya en $1968^{8}$. Durante un período de 6 años, la cirugía coronaria estableció sus fundamentos y alcanzó el reconoci- 
miento de terapéutica válida universalmente aceptada.

La cirugía coronaria es una de las mejores terapias, extensamente documentada a corto, medio y largo plazo; situándose a la cabeza del conocimiento científi$\mathrm{co}^{9}$. En Estados Unidos, el número de intervenciones quirúrgicas tuvo un incremento significativo hasta 1996 y un descenso progresivo desde el año 2000, con una reducción de 100.000 casos/año ${ }^{10}$. Esta evolución se atribuye a la introducción en 1996 de los stents que superaron al número de intervenciones quirúrgicas en 1998. En Europa también se ha detectado esta disminución significativa, a razón del 7-10\% anual, y en España el número de intervenciones se ha reducido un $16 \%$ durante el período 2000-2002 ${ }^{11}$. Este descenso contrasta con el incremento del número de intervenciones coronarias percutáneas (ICP), en torno al 15\% anual $^{12,13}$. Los avances de las ICP, incluidos los stents recubiertos con fármacos antiproliferativos, han repercutido en la reducción global de la cirugía ${ }^{14,15}$. Sin embargo, aunque en determinados grupos de pacientes los stents recubiertos han demostrado ser más efectivos, falta evidencia científica sobre resultados a largo plazo, así como en otro tipo de enfermos o de lesiones $^{16}$. Esta evolución exige adaptar la cirugía de la cardiopatía isquémica al futuro. Así en este artículo se revisan los resultados y las indicaciones y se valoran las nuevas oportunidades que incluyen la cirugía más segura, menos agresiva y asociada a otros procedimientos quirúrgicos.

\section{RESULTADOS. INDICACIONES}

\section{Resultados}

\section{Mortalidad}

La cirugía coronaria es probablemente la técnica quirúrgica con resultados mejor documentados a corto, medio y largo plazo. La mortalidad hospitalaria en Estados Unidos y Europa es inferior al 2,5\% ${ }^{17-19}$. Aproximadamente el $98 \%$ de los pacientes están vivos al mes y el 97, el 92, el 81 y el 66\% están vivos 1, 5, 10 y 15 años, respectivamente, después de la intervención ${ }^{20}$. La evolución de esta curva, con un descenso inicial pequeño durante los primeros meses, casi aplanamiento posterior hasta el quinto año y un descenso progresivo desde entonces y más evidente a partir del octavo año, está relacionada con la oclusión de los injertos, la progresión de la enfermedad y el desarrollo de comorbilidades. La revascularización con injertos arteriales mejora esta curva de supervivencia ${ }^{21,22}$.

A partir de grandes bases de datos, se han desarrollado modelos de estratificación de riesgo para predecir la mortalidad y la evolución ${ }^{18-20,22-24}$. Todas las escalas incluyen las siguientes variables como predictoras de mortalidad hospitalaria: edad, sexo femenino, reo- peración, urgencia, disfunción ventricular izquierda, cirugía asociada, insuficiencia renal, arteriopatía sintomática extracardíaca. A estos factores hay que añadir otros relacionados con la anatomía, tales como la revascularización incompleta, la proporción de vasos distales de pequeño calibre, la asociación de endarterectomía, la enfermedad severa del tronco común izquierdo y la experiencia del cirujano ${ }^{20}$. En la tabla 1 está representada la escala de predicción de riesgo quirúrgico EuroSCORE, desarrollada a partir de 19.030 pacientes, operados en Europa durante el período septiembre-diciembre de 1995.

\section{Evolución}

Después de la cirugía, la probabilidad de estar libre de angina a 1, 5, 10, 15 y 20 años es del 95, el 82, el 61 , el 38 y el $21 \%$, respectivamente ${ }^{25}$. Estos resultados demuestran que a largo plazo la angina es casi inevitable, aunque el intervalo medio desde la cirugía supera ligeramente los 12 años. El uso de la AMI para revascularizar la DA reduce la recurrencia de la angina, principalmente a partir del cuarto año ${ }^{26}$. La probabilidad de estar libre de infarto a los 30 días, 5, 10, 15 y 20 años es del 97, el 94, el 86, el 73 y el 56\%, respectivamente ${ }^{26}$. La prevalencia de muerte súbita es baja, con el 97\% de pacientes libres a los 10 años. La disfunción ventricular izquierda severa es el principal

\section{TABLA 1. Predicción de riesgo quirúrgico EuroSCORE ${ }^{19}$}

\begin{tabular}{|c|c|}
\hline Factores de riesgo & Puntos \\
\hline Edad (cada 5 años a partir de 60 años) & 1 \\
\hline Mujer & 1 \\
\hline Enfermedad pulmonar crónica & 1 \\
\hline Arteriopatía extracardíaca & 2 \\
\hline Reoperación & 3 \\
\hline Insuficiencia renal & 2 \\
\hline Endocarditis activa & 3 \\
\hline Estado preoperatorio crítico & 3 \\
\hline Angina inestable & 2 \\
\hline Fracción de eyección inferior a 0,30 & 3 \\
\hline Infarto de miocardio reciente & 2 \\
\hline Hipertensión pulmonar & 2 \\
\hline Emergencia & 2 \\
\hline Cirugía no coronaria & 2 \\
\hline Cirugía de la aorta torácica & 3 \\
\hline Comunicación interventricular postinfarto & 4 \\
\hline Índice EuroSCORE (puntos) & Mortalidad estimada (\%) \\
\hline $0-2$ & 1-0 \\
\hline $3-5$ & $2,62-3,51$ \\
\hline $6-8$ & $6,51-8,37$ \\
\hline $9-10$ & $14,0-19,0$ \\
\hline $11-13$ & $31,0-42,0$ \\
\hline$\geq 13$ & $>42,0$ \\
\hline
\end{tabular}


factor de riesgo relacionado con la muerte súbita; mientras que este riesgo es mínimo en los pacientes con fracción de eyección (FE) superior a 0,55, alcanza el $15 \%$ a los 15 años en aquellos con FE inferior a 0,25 . La implantación de un desfibrilador en este grupo con disfunción ventricular severa no mejora la supervivencia ${ }^{27}$. La cirugía coronaria no reduce la frecuencia y severidad de las arritmias ventriculares inducidas en la mayoría de los pacientes ${ }^{28}$, debido a que estas arritmias guardan más relación con cicatrices ventriculares que con áreas de isquemia. Sin embargo, existe un subgrupo de pacientes sin cicatriz o aneurisma ventricular, cuyas arritmias son secundarias a isquemia y mejoran después de la cirugía. La fibrilación auricular es un factor que incide negativamente en la supervivencia a largo plazo después de la cirugía ${ }^{20}$ y es una complicación frecuente en el postoperatorio inmediato del 23-33\% ${ }^{29,30}$ de los pacientes operados con circulación extracorpórea (CEC).

La capacidad funcional máxima mejora y esta recuperación guarda una relación directa con la FE preoperatoria y la revascularización completa ${ }^{31}$. La calidad de vida mejora hasta valores próximos a los de la población general, aunque la cuarta parte de los pacientes alcanzan solamente valores subóptimos ${ }^{32}$. La calidad de vida preoperatoria, comorbilidad, sexo femenino, edad y enfermedad cardíaca crónica son factores que inciden negativamente en la calidad de vida postoperatoria ${ }^{32}$.

\section{Reoperaciones}

La probabilidad de no requerir una reintervención coronaria, percutánea o quirúrgica, a los 30 días, 5, 10, 15 y 20 años es del 99,7, el 97, el 89, el 72 y el $48 \%$, respectivamente ${ }^{33}$. La causa más frecuente de reintervención es la aterosclerosis del injerto venoso seguida de la progresión de la enfermedad en la arteria nativa $^{34}$. Aunque el uso de la AMI no ha reducido significativamente la prevalencia de reintervenciones en la serie de Sergeant et $\mathrm{al}^{33}$, la experiencia de la mayoría de los grupos muestra que el uso de la AMI izquierda para revascularizar la DA reduce el número de reintervenciones y el intervalo entre la primera y la segunda intervención ${ }^{35,36}$. La revascularización con las 2 AMI conlleva una reducción aún mayor de reoperaciones ${ }^{22,35-37}$.

El riesgo de la reintervención duplica el riesgo de la primera intervención ${ }^{33,34}$ y está más relacionado con la mayor prevalencia de los factores de riesgo que con la técnica quirúrgica propiamente dicha ${ }^{34,38}$. Los factores que influyen negativamente en la supervivencia precoz y tardía son la enfermedad severa del tronco común o tres vasos, la edad y la disfunción ventricular izquierda severa $^{22,38}$. La ICP tiene un protagonismo creciente en los pacientes sintomáticos después de cirugía coronaria $^{34}$. Los resultados sobre los injertos venosos son significativamente mejores con stent que con angioplastia con balón: eficacia, diámetro de la luz, supervivencia, infarto de miocardio y necesidad de intervención quirúrgica ${ }^{39}$. La ICP con control de ecografía intracoronaria del tronco común izquierdo protegido es una alternativa real a la reintervención quirúrgica ${ }^{40}$.

\section{Tipo de injerto}

La vena safena interna es históricamente el injerto más utilizado. Tras su implantación, desarrolla una hiperplasia de la íntima, no progresiva e inversamente proporcional al flujo del injerto, aproximándose su calibre al del vaso nativo, lo que se considera un proceso de remodelado. El desarrollo de aterosclerosis con placas, úlceras y trombosis tiene mayor importancia; el control de los factores de riesgo y el tratamiento agresivo con antiagregantes plaquetarios y estatinas ${ }^{41}$ mejora o retrasa estos cambios. Durante el primer año de evolución, el $20 \%$ de los injertos presenta una estenosis de la anastomosis proximal con oclusión del 25\% de estos injertos antes de los 5 años; en el $50 \%$ de los injertos se puede observar cierto grado de estenosis en la anastomosis distal que en la mayoría de los casos no progresa. La permeabilidad del injerto venoso en grupos heterogéneos de pacientes es del $90 \%$ al mes y del 50 , el 30 y el $20 \%$ a los 10,15 y 20 años, respectivamente $^{35,41}$. Sin embargo, la permeabilidad de la vena safena anastomosada a la DA es del $80 \%$ a los 10 $\operatorname{años}^{35}$.

Aunque la superioridad de la AMI estaba probada desde 1973, su aceptación universal no llegó hasta mediados de la década de los ochenta. La AMI reduce la recurrencia de angina, la prevalencia de infarto de miocardio a largo plazo, las reintervenciones y el intervalo entre la primera y la segunda intervención quirúrgica $^{25,26,35}$. La permeabilidad de la AMI izquierda in situ, revascularizando la DA, es del 95 y el $90 \%$ a los 10 y 15 años, respectivamente ${ }^{22,33,35,36}$. El 5-10\% de los injertos permeables presentan estenosis, aunque en la mayoría de los casos no progresa hacia la oclusión. El bajo umbral de velocidad trombogénica, la resistencia al desarrollo de aterosclerosis, la liberación de factor de crecimiento vascular endotelial y prostaciclina, y la reactividad a los vasodilatadores explican su alta permeabilidad y su vigencia como injerto ideal ${ }^{35,36,41,42}$. Un factor que influye en la alta permeabilidad de la AMI es la revascularización de la DA. La revascularización con AMI de otras arterias diferentes de la DA, de arterias con estenosis inferiores al 60\%, o el uso de la AMI derecha como bypass aortocoronario en lugar de como injerto in situ reducen la permeabilidad de la AMI; los resultados son similares a los de la vena safena $^{41-43}$. La reserva adecuada de flujo con la AMI derecha anastomosada en Y a la AMI izquierda queda corroborada por excelentes resultados clínicos de flujo y permeabilidad ${ }^{44,45}$. La extensión del injerto es también una técnica segura con una permeabilidad mayor 
del 95\% ${ }^{46}$, así como las anastomosis secuenciales, excepto cuando se revascularizan ramas marginales de la arteria circunfleja o distales de la arteria coronaria derecha ${ }^{47}$. El uso de las dos AMI, sin incrementar la mortalidad hospitalaria, mejora la supervivencia a largo plazo y reduce la prevalencia de reintervenciones $^{22,36,37,41,45}$; estas diferencias a favor de la doble AMI son mayores en los pacientes de edad avanzada con múltiples factores de riesgo que en los pacientes jóvenes, durante los primeros 10 años postoperatorios ${ }^{41}$. Las reticencias al uso de la doble AMI en pacientes diabéticos ha quedado solventada con la disección de la AMI «esqueletizada ${ }^{48}$. El grupo de pacientes diabéticos es el que mayor beneficio obtiene del uso de la doble AMI con mayor supervivencia y reducción de la prevalencia de infarto de miocardio y necesidades de reintervención ${ }^{36}$. Estos resultados recomiendan la revascularización coronaria completa con las dos AMI en los pacientes diabéticos y aquellos que tengan una esperanza de vida mayor de 10 años o múltiples factores de riesgo asociados ${ }^{49}$.

La superioridad de la AMI sobre la vena safena reintrodujo el uso de la arteria radial en 1992, que se había abandonado a mediados de la década de los setenta. Su uso se ha extendido combinado con la AMI, como injerto aortocoronario, simple o secuencial, en Y o como extensión de la $\mathrm{AMI}^{50}$. Los resultados publicados mostraban una permeabilidad similar a la AMI derecha a medio plazo y a la AMI izquierda a corto plazo, con un $95 \%$ de los injertos permeables a los 5 años $^{50}$. Sin embargo, su uso está en rápida regresión desde el año 2004 por la recurrencia de angina asociada a espasmo y a la publicación de una permeabilidad a medio plazo inferior a la AMI y a la vena safena, con un porcentaje de oclusión o estenosis severa del $51 \%$ a los 2 años y necesidad de reintervención, percutánea o quirúrgica, en el $27 \%$ de los pacientes ${ }^{51}$. El uso de la arteria gastroepiploica y la arteria epigástrica inferior está también en regresión por la recurrencia de angina debido a la desproporción del calibre injerto-arteria coronaria y una permeabilidad estimada del 80 y el $62 \%$ a los 5 y 10 años, respectivamente ${ }^{52,53}$.

\section{Indicaciones}

La indicación de cirugía exige el estudio comparativo del beneficio sobre el tratamiento médico y la ICP. La complejidad de esta propuesta, aparentemente simple, radica en que el beneficio comparativo de la cirugía puede ser mayor en función de las circunstancias. Las predicciones y comparaciones deben ser precisas y para ello poseemos un volumen de información enorme que nos permite proponer a cada paciente el tratamiento más eficiente. Las lesiones coronarias y los factores de riesgo tienen grandes variaciones y las recomendaciones basadas en estudios con grupos heterogéneos de pacientes tienen escaso valor. Las indica- ciones generales consensuadas por el American College of Cardiology y la American Heart Association en 1999 y actualizadas en $2004^{54}$, se presentan en la tabla 2 , siguiendo el resumen de Alonso Martín et al ${ }^{13}$.

En los pacientes con cardiopatía isquémica estable, la angina ligera o moderada, corroborada por cambios ligeros reversibles con las técnicas de detección de isquemia, no es per se indicación quirúrgica o de ICP. La ICP, si la anatomía es favorable, o la intervención quirúrgica están indicadas en la angina severa. En la estenosis del tronco común izquierdo, la cirugía mejora claramente el pronóstico y la indicación es prioritaria o urgente, según la severidad de la estenosis o la sintomatología ${ }^{55}$, aunque recientemente se ha incorporado con éxito la ICP en casos seleccionados ${ }^{40,56,57}$. La intervención quirúrgica mejora la supervivencia de los pacientes con enfermedad de tres vasos y baja $\mathrm{FE}^{58}$ o FE normal $^{59}$, aunque con menor significación. En la enfermedad de dos vasos, la ICP es eficaz para muchos pacientes; el pronóstico con tratamiento médico es, generalmente, mejor que en la enfermedad de tres vasos y la intervención quirúrgica no está indicada en la mayoría de los casos. Sin embargo, cuando la lesión es equivalente de tronco común izquierdo, la lesión severa de la DA es proximal, la FE es baja o hay una gran área de miocardio viable con alto riesgo, la intervención quirúrgica está indicada ${ }^{59}$. La indicación quirúrgica en la enfermedad de un vaso es poco frecuente porque los resultados del tratamiento médico y de la ICP son buenos. El bypass de AMI a la DA es una opción apropiada para los pacientes con lesión proximal de la DA y evidencia de isquemia extensa o FE baja. En el síndrome coronario agudo sin elevación del segmento ST, la indicación de cirugía está en función del resultado de la coronariografía realizada a los pacientes con riesgo alto o moderado, según la Sociedad Española de Cardiología ${ }^{13}$.

Aunque la cirugía mejora el pronóstico de los pacientes con disfunción ventricular izquierda, el riesgo y los beneficios son inciertos en los pacientes con FE inferior a 0,30. La mortalidad hospitalaria en las décadas de los años setenta y ochenta variaba alrededor del $10 \%$, con cifras entre el $2,9 \%$ y el $39 \%$, según la presencia o no de hipertensión pulmonar, insuficiencia cardíaca, arritmias ventriculares y estudios preoperatorios de viabilidad ${ }^{60}$. Las mejoras de las técnicas anestésicas y de monitorización, la protección miocárdica con cardioplejía y la cirugía coronaria sin CEC, así como el perfeccionamiento de las técnicas de cuantificación de la isquemia y viabilidad, ofrecen a los pacientes con evidencia de miocardio hibernado una mortalidad hospitalaria quirúrgica inferior al 3\% $\%^{61,62}$. En las regiones sin potencial para la recuperación funcional, ha sido descrita recientemente una adaptación menor con hipertrofia celular miocárdica menos pronunciada, una destrucción mayor de la arquitectura miocárdica y 


\section{TABLA 2. Indicaciones de cirugía coronaria (ACC/AHA) $)^{54}$}

Clase I

Pacientes asintomáticos o con angina leve

Estenosis significativa de tronco común izquierdo ( $\geq 50 \%$ ) o equivalente ( $\geq 70 \%$ DA y Cx proximal, nivel de evidencia A)

Enfermedad significativa de 3 vasos. El beneficio es mayor con $\mathrm{FE}<0,50$ y/o grandes áreas de isquemia (nivel de evidencia $\mathrm{C}$, si angina estable nivel A)

Angina estable

Las anteriores

Enfermedad significativa de 2 vasos: a) DA proximal y/o FE $<0,50$ o isquemia demostrable (nivel de evidencia A); $b$ ) sin enfermedad de DA, pero con criterios de riesgo alto y área grande de miocardio viable (nivel de evidencia $\mathrm{B}$ )

Angina estable incapacitante con riesgo aceptable de la cirugía (nivel de evidencia B)

Angina inestable o IAM

Angina inestable o IAM sin onda $Q$ con enfermedad de tronco común izquierdo significativa o equivalente (nivel de evidencia $A$ )

Angina inestable o IAM sin posibilidad de ICP e isquemia en evolución sin respuesta a tratamiento no quirúrgico

Clase II

Pacientes asintomáticos o con angina estable

Enfermedad de DA proximal y 1-2 vasos (clase Ila). Si FE $<0,500$ isquemia extensa, indicación clase I (nivel de evidencia A)

Enfermedad de 1-2 vasos sin enfermedad de DA proximal (clase Illb). Si hay criterios de riesgo alto y área moderada 0 extensa de miocardio viable, indicación clase lla (nivel de evidencia B)

Angina inestable o IAM sin onda $Q$

Enfermedad de DA proximal y de 1-2 vasos (clase Ila, nivel de evidencia A)

Enfermedad de 1-2 vasos sin lesión de DA proximal si ICP no es posible (clase Ilb, nivel de evidencia B)

Clase III

Angina estable con lesiones coronarias $<50 \%$ (nivel de evidencia B) o con lesiones del 50-60\% sin afectación del tronco común izquierdo e isquemia no demostrable (nivel de evidencia B) o con enfermedad de 1-2 vasos sin enfermedad de DA proximal y no evidencia de isquemia o pequeña área de miocardio viable (nivel de evidencia $B$ )

ACC: American College of Cardiology; AHA: American Heart Association; DA: arteria descendente anterior; CX: arteria circunfleja; IAM: infarto agudo de miocardio; ICP: intervención coronaria percutánea; FE: fracción de eyección.

un grado mayor de expresión genética antiapoptosis. Estos hallazgos sugieren la realización de una biopsia endomiocárdica a los pacientes con estudios de viabilidad desfavorables, para indicar cirugía coronaria o trasplante cardíaco ${ }^{61}$.

\section{Indicaciones: cirugía frente a la intervención coronaria percutánea}

La elección entre cirugía e ICP en la enfermedad coronaria multivaso está condicionada por: $a$ ) la elección entre las dos técnicas será posible únicamente en el subgrupo de pacientes en los que sea posible realizar una revascularización más o menos completa con $\mathrm{ICP}^{13}$. La cirugía coronaria se puede realizar sobre arterias de pequeño calibre y en la serie de Levin et al ${ }^{63}$ se pudo implantar injertos con éxito al $73 \%$ de los vasos considerados inadecuados por pequeño calibre distal o ausencia de llenado en la coronariografía; $b$ ) si el riesgo del procedimiento es similar, aunque la prevalencia de reestenosis y la necesidad de reintervención sea mayor después de la ICP y con independiencia de los estudios de costes, es obvio que el paciente y su cardiólogo aceptarán la ICP porque siempre será menos agresiva y se puede repetir indefinidamente.

Durante la década de los noventa fueron publicados los resultados de 9 estudios aleatorizados que comparaban la ICP y la cirugía; sus conclusiones fueron ob- jeto de un documento conjunto publicado por el American College of Cardiology y la American Heart Association ${ }^{64}$. Los resultados de estos estudios mostraron diferencias significativas a favor de la cirugía en cuanto a recurrencia de angina y necesidad de nueva revascularización coronaria, sin diferencias en la mortalidad, excepto en el BARI que la supervivencia a los 7 años fue mejor después de cirugía, a expensas de los pacientes diabéticos ${ }^{13,64,65}$. Estos resultados se han corroborado en el ARTS ${ }^{66}$, el SoS $^{67}$ y el SIMA ${ }^{68}$. El desarrollo de los stents recubiertos puede reducir o suprimir las ventajas de la cirugía en la necesidad de nuevos procedimientos de revascularización ${ }^{14-16}$, como lo sugieren los resultados iniciales del ARTS II $^{13}$ y sin que se hayan presentado aún los resultados preliminares del Syntax-Trial ${ }^{69}$.

\section{DÓNDE ESTAMOS Y HACIA DÓNDE VAMOS. NUEVOS RETOS Y OPORTUNIDADES}

La mayoría de los cirujanos asumieron que el futuro debe ser como el pasado y la edad de oro para una industria no es garantía de supervivencia a largo plazo. El futuro está ligado a un ciclo innovador que adapte la cirugía a las necesidades de la población, las nuevas tecnologías y las actividades pioneras. El éxito dependerá de la actitud innovadora de los cirujanos, el apoyo de la industria y de los gestores de la sanidad, pero fundamentalmente de los pacientes. 


\section{Progreso y nuevas tecnologías}

El desarrollo de la cirugía cardíaca se ha acompañado de unos progresos técnicos, gracias a los cuales el cirujano se rodea ahora de mayores garantías. La introducción de la CEC abrió la puerta a una multitud de posibilidades terapéuticas y ello contrasta con diversas actitudes que pretenden subestimar el valor real de la CEC.

\section{Progresos técnicos}

Los pacientes intervenidos actualmente tienen lesiones coronarias más severas y difusas, hay una mayor proporción de enfermos con disfunción ventricular y comorbilidades, así como un aumento de reoperaciones. El aumento del riesgo de mortalidad estima$\mathrm{da}^{18,19,23}$ se asocia a una reducción de la mortalidad realmente observada. Esta paradoja se debe a que los modelos de estratificación de riesgo sobrestiman la mortalidad, porque pacientes que fallecían cuando se desarrollaron estas escalas actualmente sobreviven, y un subgrupo del 5-6\% genera estancias prolongadas en cuidados intensivos y un incremento de los costes. Esta cirugía más segura se ha conseguido por la experiencia creciente; el mejor conocimiento de la bioquímica, fisiología y fisiopatología, y el desarrollo paralelo de otras especialidades y los progresos en la tecnología complementaria:

- Técnicas diagnósticas y monitorización. El fracaso en cirugía cardíaca es la consecuencia de fallos técnicos o de falta de conocimiento. El mejor conocimiento preoperatorio mejora la indicación y ofrece una información más precisa del riesgo/beneficio de la cirugía. En cirugía coronaria destaca el desarrollo tecnológico para valorar la viabilidad miocárdica mediante ecocardiografía, gated-SPECT, tomografía de emisión de positrones y resonancia magnética cardíaca que nos ofrecen información precisa sobre la reserva contráctil, la microperfusión miocárdica, la integridad celular y el metabolismo celular ${ }^{70}$. La monitorización intraoperatoria ofrece una información más precisa del estado hemodinámico y medio interno, a través de los nuevos catéteres de SwanGanz, la ecocardiografía, los nuevos equipos de exploración analítica y los desarrollos tecnológicos de monitorización y seguridad de las bombas de CEC. La medida intraoperatoria del flujo de los injertos con ultrasonidos es obligatoria, especialmente en la cirugía coronaria sin CEC, cuantificando el flujo, su reserva y el índice de pulsatilidad que nos indica la calidad de la anastomosis ${ }^{71}$. El seguimiento con tomografía computarizada (TC) multicorte ha sido objeto de numerosos estudios que muestran una sensibilidad del $86-97 \%$ y una especificidad del $89-100 \%$ para determinar oclusión de injertos ${ }^{72,73}$. La medida intraoperatoria de flujos y el seguimiento con TC multicorte ofrecen dos herramientas excelentes para desarrollar estudios comparativos de las técnicas de cirugía coronaria.

- Protección miocárdica. La incorporación de la cardioplejía a finales de la década de los setenta cambió el desarrollo de la cirugía cardíaca. Los progresos de la protección miocárdica han permitido abordar con excelentes resultados la cirugía en pacientes con disfunción ventricular izquierda. Sin embargo, no podemos considerar que el problema esté resuelto. El 65\% de las muertes postoperatorias son por causas cardíacas y la protección miocárdica defectuosa desempeña un papel preponderante. Por otra parte, las lesiones miocárdicas intraoperatorias, expresadas por elevación enzimática, infarto o bajo gasto ejercen un efecto negativo sobre la supervivencia a largo plazo $^{74}$. Hay principios demostrados que deben ser respetados: $a$ ) el mantenimiento de la CEC en normotermia o ligera hipotermia. La hipotermia inferior a $32{ }^{\circ} \mathrm{C}$ es inútil y potencialmente dañina; $b$ ) la superioridad de la cardioplejía hemática sobre la cristaloide; $c$ ) el impacto de la CEC sobre la protección miocárdica con la liberación de mediadores inflamatorios con efectos inotrópicos negativos. A estos principios se han sumado controversias técnicas que han mostrado resultados comparables: cardioplejía caliente o a $4{ }^{\circ} \mathrm{C}$, perfusión continua o discontinua, vía anterógrada o retrógrada ${ }^{74}$. Varias propuestas que produjeron entusiasmo, como la adición de L-arginina, moléculas antioxidantes, insulina, inhibidores del intercambio sodio-protones, filtros leucocitarios o el preacondicionamiento isquémico, no han conseguido la corroboración clínica ${ }^{74,75}$. Diferentes alternativas están actualmente en marcha, pendientes de resultados clínicos, como la adición a la cardioplejía de esmolol o de anticuerpos monoclonales inhibidores del complemento ${ }^{74}$.

- Anestesia y cuidados. El fast track es una atención intraoperatoria específica que lleva a un estado postoperatorio mejorado, reduciendo la necesidad de soporte respiratorio, cardíaco y de cuidados intensivos. La atención intraoperatoria puede dividirse en 4 apartados: el cerebro, el calentamiento, el tono vascular periférico y el cuidado meticuloso del paciente. Los nuevos fármacos anestésicos de acción más rápida hacen mucho más fácil el desarrollo de esta estrategia y la anestesia epidural torácica facilita la extubación inmediata, protege mejor la función pulmonar y cardíaca, y reduce el dolor postoperatorio ${ }^{76}$. Nuevos inotrópicos como el levosimendan, el conocimiento de los beneficios cardioprotectores del isoflurano y de las hormonas tiroideas han mejorado la seguridad. Las técnicas de autotransfusión, el desarrollo de los recuperadores sanguíneos, el mejor conocimiento de la hemostasia, el uso de antifibrinolíticos y la cirugía sin CEC han reducido el consumo de hemoderivados. Estos progresos han llevado a desarrollar recientemente la cirugía coronaria ambulatoria $^{77}$. Para obtener los beneficios esperados de estos 
progresos, es necesario un proceso activo que requiere una gestión intraoperatoria específica.

\section{Cirugía coronaria menos invasiva}

Los progresos de la anestesia se han complementado con el desarrollo de técnicas quirúrgicas menos agresivas, a través de una industria dispuesta a invertir. La cirugía coronaria miniinvasiva es equivalente a cirugía sin CEC y puede realizarse a través de pequeñas incisiones o bien por esternotomía media:

- Acceso por miniincisión. Denominada en la terminología anglosajona MIDCAB, produjo perplejidad en la década de los noventa por las numerosas incisiones que se inventaron; en $\langle\mathrm{S} »,\langle\mathrm{~L} »,\langle\mathrm{~J} »,\langle\mathrm{C} »,\langle\mathrm{~T} »$, etc. Actualmente sus indicaciones se limitan al número reducido de casos de revascularización con AMI de lesiones únicas de la DA con abordaje por minitoracotomía izquierda, miniesternotomía inferior o a revascularización con arteria gastroepiploica de la arteria descendente posterior con abordaje subxifoideo ${ }^{78}$. La revascularización híbrida, basada en la demostración de que el factor más determinante de supervivencia a largo plazo es la revascularización de la DA con AMI, consiste en la revascularización con AMI de la DA e ICP en el resto de las lesiones. La constancia de que la agresión quirúrgica está relacionada principalmente con la CEC limita sus indicaciones a pacientes con alta probabilidad de cirugía posterior; aquellos con lesiones más críticas de la DA, el resto de las arterias susceptibles de ICP y alto riesgo, y en casos de lesión severa de tronco común y alto riesgo.

- Cirugía coronaria sin CEC por esternotomía. La agresividad de la cirugía coronaria está relacionada con la CEC y no con el tamaño de la incisión. Esto unido a la importancia de la revascularización completa que se puede conseguir por esternotomía media sin CEC, mediante el uso de estabilizadores coronarios, ha derivado la atención hacia la cirugía coronaria sin CEC con revascularización completa por esternotomía media, denominada en la nomenclatura anglosajona OPCAB. La cirugía coronaria sin CEC reduce, aunque no suprime, los valores circulantes de mediadores inflamatorios ${ }^{74}$, y la eliminación del pinzamiento aórtico reduce el sufrimiento miocárdico. Aunque los resultados clínicos no han alcanzado aún evidencia clase I, los resultados de series amplias observacionales indican el beneficio de esta técnica en términos de supervivencia a corto y largo plazo, principalmente en pacientes con disfunción ventricular, diabéticos y ancianos ${ }^{79-81}$, incidencia de infarto de miocardio y accidente cerebrovascular ${ }^{82}$, y reducción de la incidencia de fibrilación auricular postoperatoria $^{83}$. La cirugía coronaria con injertos arteriales sin CEC asociada a anestesia epidural torácica y fast track representa actualmente el patrón oro. Aunque actualmente representa la cuarta parte de la cirugía coronaria europea, a expensas principalmente de los equipos que han decidido realizar la conversión, su aplicación se incrementará por los progresos técnicos que facilitan las anastomosis ${ }^{84}$ y la formación creciente de cirujanos. Un nuevo abordaje es la cirugía coronaria con circuito cerrado y simplificado de $\mathrm{CEC}^{85}$ que produce una agresión quirúrgica intermedia entre la cirugía con CEC convencional y la cirugía sin CEC. Sin embargo, su utilidad está en duda porque para los equipos que han realizado la conversión a cirugía sin CEC, ésta representa más del $90 \%$ de la cirugía coronaria aislada total ${ }^{9}$.

- Cirugía endoscópica y robótica. La cirugía endoscópica con instrumentación convencional está limitada por la imprecisión de las suturas secundaria a la visualización bidimensional y se ha reducido a la disección de la AMI o la vena safena. La cirugía robótica con telemanipulación asistida por ordenador y visión tridimensional está en las fases iniciales de aplicación clínica $^{86}$.

\section{Cirugía asociada}

La cirugía en una población cada vez más anciana ha incrementado la asociación de otros procedimientos:

- Cirugía valvular. En la base de datos STS-Database, el 48,2\% de la cirugía valvular realizada en Estados Unidos durante el período 1992-2001 estuvo asociada a cirugía coronaria ${ }^{18}$.

La mayoría de los pacientes con cirugía coronaria asociada a sustitución valvular aórtica tiene una estenosis degenerativa y una edad superior a los 65 años. En este grupo, la decisión de un abordaje agresivo para la estenosis aórtica moderada es más agresiva porque la tolerancia a la reoperación es menor y las bioprótesis en este grupo de edad tienen una durabilidad excelente $^{87}$.

La presencia de una insuficiencia mitral isquémica se asocia a una mortalidad elevada, directamente relacionada con el grado de regurgitación ${ }^{88}$ y la cirugía coronaria aislada no corrige significativamente la insuficiencia mitral ${ }^{89}$. En la insuficiencia mitral de la cardiopatía isquémica pueden coexistir los tres tipos funcionales de la clasificación de Carpentier. La restricción de la apertura de las valvas (tipo IIIb), secundaria a la acinesia o discinesia ventricular, incrementa la distancia entre los músculos papilares y el anillo mitral, la excesiva tracción de las cuerdas impide la coaptación de las valvas, la dilatación ligera o moderada del anillo (tipo I) está frecuentemente asociada y el prolapso valvular (tipo II) está presente en un tercio de los $\operatorname{casos}^{90}$. La anuloplastia restrictiva mitral asociada a cirugía coronaria tiene una mortali- 
dad hospitalaria del 5\% en pacientes con FE inferior a 0,35 y una supervivencia a los 2 años del $84 \%$, con mejoría significativa de los volúmenes ventriculares $^{91}$.

- Remodelado ventricular. El remodelado del ventrículo izquierdo postinfarto es un fenómeno complejo y la ICP temprana protege el subepicardio pero tiene menos éxito en la recuperación del subendocardio. Alrededor del $30 \%$ de los pacientes tratados con éxito con ICP primaria presentan a los 6 meses un aumento del volumen diastólico de ventrículo izquierdo superior al $20 \%{ }^{92}$. Los estudios no aleatorizados que han comparado los resultados entre tratamiento médico y quirúrgico en pacientes con disfunción ventricular e insuficiencia cardíaca inclinan la balanza a favor del tratamiento quirúrgico ${ }^{92}$. Sin embargo, para que el tratamiento quirúrgico sea eficaz, deben tenerse en cuenta la revascularización completa, la reducción del volumen ventricular a expensas del tabique interventricular y la restauración de la geometría del ventrículo recuperando la forma elíptica ${ }^{92}$. Si los resultados confirman las hipótesis del estudio $\mathrm{STICH}^{93}$, la cirugía de las secuelas del infarto de miocardio recuperará el protagonismo perdido.

- Cirugía asociada de la fibrilación auricular. La supervivencia a largo plazo después de cirugía coronaria es significativamente menor en los pacientes con fibrilación auricular crónica ${ }^{20}$ y la prevalencia de fibrilación auricular postoperatoria es un factor limitante al desarrollo de la cirugía ambulatoria ${ }^{77}$. El desarrollo de catéteres bipolares de radiofrecuencia simplifica la ablación epicárdica, mejora la transmuralidad y elimina la posibilidad de perforación esofágica, extendiendo las indicaciones de la cirugía asociada coronariaablación como tratamiento del pequeño porcentaje de pacientes operados de cirugía coronaria en fibrilación auricular o como prevención asociada a la cirugía menos agresiva de corta estancia.

\section{CONCLUSIONES}

La cirugía coronaria viene avalada por los excelentes resultados, bien documentados a largo plazo, como consecuencia de la revascularización completa y la utilización de una o dos AMI. La agresión quirúrgica se ha reducido con la cirugía coronaria sin CEC, el uso de la anestesia epidural torácica y la introducción de la cirugía ambulatoria. El descenso progresivo de la cirugía en favor de la ICP exige el desarrollo de estrategias ligadas a un ciclo innovador que adapte la cirugía a las necesidades de la población, las nuevas tecnologías y a las actividades pioneras. El éxito de este ciclo innovador dependerá de la actitud de los cirujanos, el apoyo inversor de la industria, de los gestores de la sanidad, pero fundamentalmente de los pacientes.

\section{BIBLIOGRAFÍA}

1. Mueller RL, Rosengart TK, Isom OW. The history of surgery of ischemic heart disease. Ann Thorac Surg. 1997;63:869-78.

2. Kolesov VI, Potashov LV. Surgery of coronary arteries. Eksp Khir Anesteziol. 1965;10:3-8.

3. Garrett HE, Dietrich EB, DeBakey ME. Myocardial revascularization. Surg Clin N Am. 1966;46:863-71.

4. Favaloro RG. Saphenous vein graft in the surgical treatment of coronary artery disease: operative technique. J Thorac Cardiovasc Surg. 1969;58:178-85.

5. Loop FD, Cosgrove DM, Lytle BW, Thurer RL, Simpferndorfer C, Taylor PC, et al. An 11 year evolution of coronary arterial surgery (1968-1978). Ann Surg. 1979;190:444-50.

6. Green GE, Stertzer SH, Reppert EH. Coronary arterial bypass graft. Ann Thorac Surg. 1968;5:443-50.

7. Johnson WD, Flemma RJ, Lepley D Jr, Ellison EH. Extended treatment of severe coronary artery disease: a total surgical approach. Ann Surg. 1969;170:460-70.

8. Hannan EL, Burke J. Effect of age on mortality in coronary artery bypass surgery in New York, 1991-1992. Am Heart J. 1994;128: 1184-91.

9. Sergeant P. The future of coronary bypass surgery. Eur J Cardiothorac Surg. 2004;26 Suppl:S4-7.

10. Cosgrove, D. View from North America's cardiac surgeons. Eur J Cardiothorac Surg. 2004;26 Suppl:S27-31.

11. Igual A, Saura E. Cirugía cardiovascular en España en el año 2002. Registro de Intervenciones de la Sociedad Española de Cirugía Cardiovascular. Cir Cardiovasc. 2004;11:97-108.

12. López Palop R, Moreu J, Fernández Vazquez F, Hernandez R. Registro Español de Hemodinámica y Cardiología Intervencionista. XIII Informe Oficial de la Sección de Hemodinámica y Cardiología Intervencionista de la Sociedad Española de Cardiología (1990-2003). Rev Esp Cardiol. 2004;57:1076-89.

13. Alonso Martín JJ, Curcio Ruigómez A, Cristóbal Varela C, Tarín Vicente MN, Serrano Antolín JM, Talavera Calle P, et al. Indicaciones de revascularización: aspectos clínicos. Rev Esp Cardiol. 2005;58:198-216.

14. Oliva G, Espallargues M, Pons JM. Stents con drogas antiproliferativas: revisión sistemática de los beneficios y estimación del impacto socioeconómico. Rev Esp Cardiol. 2004;57:123-9.

15. Alfonso F, Bermejo J, Segovia J. Estado actual de la revascularización coronaria. Rev Esp Cardiol. 2005;58:194-7.

16. Lázaro P. Stents recubiertos de fármacos: eficacia, efectividad, eficiencia y evidencia. Rev Esp Cardiol. 2004;57:608-12.

17. Sergeant P, Blackstone E, Meyns B. Can the outcome of coronary bypass grafting be predicted reliably? Eur J Cardiothorac Surg. 1997;11:2-9.

18. STS National Database. Unadjusted isolated CABG operation mortality. Disponible en: www.ctsnet.org/doc/5408-5410

19. Roques X, Nashef SAM, Gauducheau ME. Risk factors and outcome in European cardiac surgery: analysis of the EuroSCORE multinational database of 19,030 patients. Eur J Cardiothorac Surg. 1999;15:816-23.

20. Sergeant P, Blackstone E, Meyns B. Validation and interdependence with patient-variables of the influence of procedural variables on early and late survival after CABG. K.U. Leuven Coronary Surgery Program. Eur J Cardiothorac Surg. 1997;12: 1-19.

21. Cameron A, Davis KB, Green G, Schaff HV. Coronary bypass surgery with internal-thoracic artery grafts-effects on survival over a 15-year period. N Engl J Med. 1996;334:216-9.

22. Lytle BW, Blackstone EH, Loop FD, Houghtaling PL, Arnold $\mathrm{JH}$, Akhrass R, et al. Two internal thoracic artery grafts are better than one. J Thorac Cardiovasc Surg. 1999;117:855-72.

23. Parsonnet V, Bernstein AD, Gera M. Clinical usefulness of riskjustified outcome analysis in cardiac surgery in New Jersey. Ann Thorac Surg. 1996;61 Suppl:S8-11.

24. Jones RH, Hannan EL, Hammermeister KE, Delong ER, O'Connor GT, Luepker RV, et al. Identification of preoperative varia- 
bles to risk adjustment of short-term mortality alters coronary artery bypass graft surgery. The Working Group Panel on the Cooperative CABG Database Project. J Am Coll Cardiol. 1996;28:1478-87.

25. Sergeant $\mathrm{P}$, Blackstone E, Meyns B. Is return of angina after coronary artery bypass grafting immutable, can it be delayed, and is it important? J Thorac Cardiovasc Surg. 1998;116:44053.

26. Sergeant P, Blackstone E, Meyns BP. Does arterial revascularization decrease the risk of infarction after coronary artery bypass grafting? Ann Thorac Surg. 1998;66:1-11.

27. Bigger JT Jr. Prophylactic use of implant cardiac defibrillators in patients at high risk for ventricular arrythmia. Patch Trial Investigators. N Engl J Med. 1997;337:1569-75.

28. Guinn GA, Mathur VS. Surgical versus medical treatment for stable angina pectoris: prospective randomized study with 1 to 4 year follow-up. Ann Thorac Surg. 1976;22:524-9.

29. Maisel WH, Rawn JD, Stevenson WG. Atrial fibrillation after cardiac surgery. Ann Intern Med. 2001;135:1061-73.

30. Hogue CW Jr, Hyder ML. Atrial fibrillation after cardiac operation: risks, mechanisms, and treatment. Ann Thorac Surg. 2000; 69:300-6.

31. Hossack KF, Bruce RA, Ivey TD, Kusumi F. Changes in cardiac functional capacity after coronary bypass surgery in relation to adequacy of revascularization. J Am Coll Cardiol. 1984;87: 901-7.

32. Permanyer Miralda C, Brotons Cuixart C, Ribera Solé A, Alonso Caballero J, Cascant Castelló P, Moral Peláez I. Resultados después de cirugía coronaria: determinantes de calidad de vida relacionada con la salud preoperatoria. Rev Esp Cardiol. 2001;54:607-16.

33. Sergeant P, Blackstone E, Meyns B, Stockman B, Jashari R. First cardiological or cardiosurgical re-intervention for ischemic heart disease after primary coronary artery grafting. Eur J Cardiothorac Surg. 1998;14:480-7.

34. Loop FD, Lytle BW, Cosgrove DM, Woods EL, Stewart RW, Golding LA, et al. Reoperation for coronary atherosclerosis. Changing practice in 2509 consecutive patients. Ann Surg. 1990;212:378-84.

35. Loop FD, Lytle BW, Cosgrove DM, Stewart RW, Goormastic M, Williams GW, et al. Influence of the internal mammary artery on 10-year survival and other cardiac events. N Engl J Med. 1986;314:1-6.

36. Stevens LM, Carrier M, Perrault LP, Hebert Y, Cartier R, Bouchard D, et al. Single versus bilateral internal thoracic artery grafts with concomitant saphenous vein grafts for multivessel coronary artery bypass grafting: effects on mortality and event-free survival. J Thorac Cardiovasc Surg. 2004;127:1408-15.

37. Rizzoli G, Schiavon L, Bellini P. Does the use of bilateral internal mammary artery (IMA) grafts provide incremental benefit relative to the use of of a single IMA? Eur J Cardiothorac Surg. 2002;22:781-6.

38. Noyez L, Van Eck FM. Long-term cardiac survival alter reoperative coronary artery bypass grafting. Eur J Cardiothorac Surg. 2004;25:59-64.

39. Savage MP, Douglas JS Jr, Fischman DL, Pepine CJ, King SB, Werner JA, et al. Stent placement compared with balloon angioplasty for obstructed coronary bypass grafts. Saphenous Vein De Novo Trial Investigators. N Engl J Med. 1997;337:740-6.

40. Soriano Triguero J. Intervencionismo percutáneo en la enfermedad del tronco común izquierdo: ¿es hora de cambiar las guías de actuación? Rev Esp Cardiol. 2004;57:1009-13.

41. Buxton B, Tatoulis J. Conduits for coronary surgery. En: Wheatley DJ, editor. Surgery of coronary artery disease. London: Arnold Publishers; 2003. p. 250-69.

42. Takeuchi K, Sakamoto S, Nagayoshi Y, Nishizawa H, Matsubara J. Reactivity of human internal thoracic artery to vasodilators in coronary artery bypass grafting. Eur J Cardiothorac Surg. 2004;26:956-9.
43. Buxton BF, Ruengsakulrach P, Fuller J, Rosalion A, Reid CM, Tatoulis J. The right internal thoracic artery graft - benefits of grafting the left coronary system and native vessels with a high grade stenosis. Eur J Cardiothorac Surg. 2000;18:255-61.

44. Markwith T, Hennen B, Scheller B, Schäefers HJ, Wendler O. Flow wire measurements after complete arterial coronary revascularization with T-grafts. Ann Thorac Surg. 2001;71:788-93.

45. Calfiore AM, Contini M, Vitolla G, Di Mauro M, Mazzei V, Teodori $\mathrm{G}$, et al. Bilateral internal thoracic artery grafting: long-term clinical and angiographic results of in situ versus Y-grafts. J Thorac Cardiovasc Surg. 2000;120:990-6.

46. Vitolla G, Giammarco G, Teodori G, Mazzei V, Canosa C, Di Mauro M, et al. Composite lengthened arterial conduits: longterm angiographic results of an uncommon surgical strategy. J Thorac Cardiovasc Surg. 2001;122:687-90.

47. Dion R, Glineur D, Derouck D, Verhelst R, Noirhomme P, El Khoury G, et al. Long-term clinical and angiographic folow-up of sequential internal thoracic artery grafting. Eur J Cardiothorac Surg. 2000;14:407-14.

48. Lytle BW. Skeletonized internal thoracic artery grafts and wound complications. J Thorac Cardiovasc Surg. 2001;121:625-7.

49. Calafiore AM, Di Giammarco GD, Teodori G, Di Mauro M, Iaco AL, Bivona A, et al. Late results of first myocardial revascularization in multiple vessel disease: single versus bilateral mammary artery with or without saphenous vein grafts. Eur J Cardiothorac Surg. 2004;26:542-8.

50. Acar C, Ramsheyi A, Pagny JY, Jebara V, Barrier P, Fabiani JN, et al. The radial artery for coronary artery bypass grafting: clinical and angiographic results at five years. J Thorac Cardiovasc Surg. 1998;116:981-9.

51. Khot UM, Friedman DT, Pettersson G, Smedira NG, Li J, Ellis SG. Radial artery bypass grafts have an increased occurrence of angiographically severe stenosis and occlusion compared with left internal mammary arteries and saphenous vein grafts. Circulation. 2004;109:2086-91.

52. Suma H, Wanibuchi Y, Terada Y, Fukuda S, Takayama S, Furuta $\mathrm{S}$. The right gastro-epiploic artery graft: clinical and angiographic mid-term results in 200 patients. J Thorac Cardiovasc Surg. 1993;105:615-23.

53. Calafiore AM, Di Giammarco G, Teodori G, D'Annunzio E, Vitolla G, Fino C, et al. Radial artery and inferior epigastric artery in composite grafts: improved mid-term angiographic results. Ann Thorac Surg. 1995;60:517-24.

54. Eagle KA, Guyton RA, Davidoff R, Edwards FH, Ewy GA, Gardner TJ, et al. Guideline Update for Coronary Artery Bypass Graft Surgery: a report of the American College of Cardiology/ American Heart Association Task Force on Practice Guidelines (Committee to Update the 1999 Guidelines for Coronary Artery Bypass Graft Surgery). American College of Cardiology. Disponible en: www.acc.org/clinical/guidelines/ cabg/index.pdf

55. Eleven-year survival in the Veterans Administration randomized trial of coronary bypass surgery for stable angina: The Veterans Administration Coronary Artery Bypass Surgery Cooperative Study Group. N Engl J Med. 1984;311:1333-9.

56. Martí V, Planas F, Cotes C, García J, Guiteras P, López L, et al. Resultados inmediatos y a largo plazo de la angioplastia con stent del tronco común. Rev Esp Cardiol. 2004;57:1029-34.

57. López-Palop R, Pinar E, Saura D, Pérez-Lorente F, Lozano I, Teruel $\mathrm{F}$, et al. Resultados a corto y medio plazo del intervencionismo coronario percutáneo sobre el tronco coronario común izquierdo no protegido en pacientes malos candidatos para revascularización quirúrgica. Rev Esp Cardiol. 2004;57:1035-44.

58. Aldermen EL, Bourassa MG, Cohen LS, Davis KB, Kaiser GG, Killop T, et al. Ten-year follow-up of survival and myocardial infarction in the randomized Coronary Artery Surgery Study. Circulation. 1990;82:1629-46.

59. Varnauskas E. Twelve-year follow-up of survival in the randomized European Coronary Surgery Study. N Engl J Med. 1988;319: $332-7$. 
60. Herreros J. Indicaciones de la revascularización quirúrgica miocárdica en la cardiopatía isquémica. Rev Clin Esp. 2002;202: 96-9.

61. Hausmann H, Meyer R, Siniawski H, Pregla R, Gutberlet M, Amthauer $\mathrm{H}$, et al. Factor exercising an influence on recovery of hibernating myocardium after coronary bypass grafting. Eur J Cardiothorac Surg. 2004;26:89-95.

62. Goldstein DJ, Beauford RB, Luk B, Karanam R, Prendergast T, Sardari F, et al. Multivessel off-pump revascularization in patients with severe left ventricular dysfunction. Eur J Cardiothorac Surg. 2003;24:72-80.

63. Levin DC, Cohn LH, Koster JK Jr, Collins JJ Jr. Accuarracy of angiography in predicting quality and caliber of the distal coronary artery lumen in preparation for bypass surgery. Circulation. 1982;66 Suppl II:II93-II6.

64. Eagle KA, Guyton RA, Davidoff R, Ewy GA, Fonger J, Gardner TJ, et al. ACC/AHA Guidelines for Coronary Artery Bypass Graft Surgery: Report of the American College of Cardiology/ American Heart Association Task Force on Practice Guidelines (Committee to Revise the 1991 Guidelines for Coronary Artery Bypass Graft Surgery). American College of Cardiology/American Heart Association. J Am Coll Cardiol. 1999;34:1262-347.

65. Seven-year outcome in the Bypass Angioplasty Revascularization Investigation (BARI) by treatment and diabetic status. J Am Coll Cardiol. 2000;35:1122-9.

66. Serruys PW, Unger F, Sousa JE, Jatene A, Bonnier HJ, Schonberger JR, et al; for the Arterial Revascularization Therapies Study Group. Comparison of coronary-artery bypass surgery and stenting for the treatment of multivessel disease. $\mathrm{N}$ Engl $\mathrm{J}$ Med. 2001;344:1117-24.

67. SoS Investigators. Coronary artery bypass surgery versus percutaneous coronary intervention with stent implantation in patients with multivessel coronary artery disease (the Stent or Surgery trial): a randomised controlled trial. Lancet. 2002;360:965-70.

68. Goy JJ, Kaufmann U, Goy-Eggenberger D, Garacheami A, Hurni $\mathrm{M}$, Carrel T, et al. A prospective randomized trial comparing stenting to internal mammary artery grafting for proximal isolation de novo left anterior coronary artery stenosis: the SIMA trial. Stenting vs Internal Mammary Artery. Mayo Clin Proc. 2000;75:1116-23.

69. Mohr F, Serruys P. Syntax Trial. Boston Scientific Corporation. Disponible en: http://www.ptca.org/press_rel/2005412pr_boston.html

70. Jiménez Borreguero LJ, Ruiz-Salmerón R. Valoración de la viabiliad miocárdica en pacientes prerrevascularización. Rev Esp Cardiol. 2003;56:721-33.

71. Mandarino W, Gorcsan J, Katz WE, Cohen HJA, Griffith BP, Zenati M. Intraoperative analysis of left mammary artery flow patterns following minimally invasive coronary bypass. J Am Coll Cardiol. 1998;31:464-5.

72. Ropers D, Ulzheimer S, Wenkel E, Baum U, Giesler T, Derlien $\mathrm{H}$, et al. Investigation of aortocoronary bypass grafts by multislice spiral computed tomography with electrocrdiographic-gated image reconstruction. Am J Cardiol. 2001;88:792-5.

73. Silber S, Finsterer S, Krischke I, Lochow P, Muhling H. Non invasive angiography of coronary bypass grafts with cardio-CT in a cardiology practice. Herz. 2003;89:167-72.

74. Menasché P. Protection myocardique: le point en 2003. J Chir Thorac Cardiovasc. 2003;7:138-41.

75. Nicolini F, Beghi C, Muscari C, Agostinelli A, Budillon AM, Spaggiari I, et al. Myocardial protection in adult cardiac surgery: current options and future challenges. Eur J Cardiothorac Surg. 2003;24:986-93.
76. Prieto I, Olivier JF, Basile F, Le N, Hemmerling TM. Immediate extubation after cardiac surgery as a routine method: first experience after 275 patients [abstract]. J Chir Thorac Cardiovasc. 2004;8 Suppl:26.

77. Bolton JWR. Ambulatory cardiac surgery. Eur J Cardiothorac Surg. 2004;26 Suppl:S68-71.

78. Niinami HN, Ogasawara H, Suda Y, Takeuchi Y. Single-vessel revascularization with minimally invasive direct coronary artery bypass. Chest. 2005;127:47-54.

79. Calafiore AM, Di Mauro M, Canosa C, Di Giammarco GD, ICAO AL, Contini M. Myocardial revascularization with and without cardiopulmonary bypass: advantages, disadvantages and similarities. Eur J Cardiothorac Surg. 2003;24:953-60.

80. Sergeant P, Wouters P, Meyns B, Bert B, Van Hemelrijck J, Bogaerts $\mathrm{C}$, et al. OPCAB versus early mortality and morbidity: an issue between clinical relevance and statistical significance. Eur J Cardiothorac Surg. 2004;25:779-85.

81. Magee MJ, Coombs LP, Peterson ED, Mack MJ. Patient selection and current practice strategy for off-pump coronary artery bypass surgery. Circulation. 2003;108 Suppl II:II9-14.

82. Van der Heijden GJMG, Nathoe HM, Jansen EWL, Grobbe DE. Meta-analysis on the effect of pump coronary bypass surgery. Eur J Cardiothorac Surg. 2004;26:81-4.

83. Raja SG, Behranwala AA, Dunning BJ. Does off-pump coronary artery surgery reduce the incidence of postoperative atrial fibrillation? Interact Cardiovasc Thorac Surg. 2004;3:647-52.

84. Tozzi P, Corno AF, Von Segesser L. Sutureless coronary anastomoses: revival of old concepts. Eur J Cardiothorac Surg. 2002;22:565-70.

85. Fromes Y, Gaillard D, Ponzio O, Chauffert M, Gerhardt MF, Deleuze $\mathrm{P}$, et al. Reduction of the inflammatory response following coronary bypass grafting with total minimal extracorporeal circulation. Eur J Cardiothorac Surg. 2002;22:527-33.

86. Reuthebuch O, Comber M, Grünenfelder J, Zünd G, Turina M. Experiences in robotically enhaced IMA-preparation as initial step towards totally endoscopic coronary artery bypass grafting. Cardiovasc Surg. 2003;11:483-7.

87. Akins CW, Hilgenberg AD, Vlahakes GJ, MacGillivray TE, Torchiana DF, Masden JC. Results of bioprosthetic versus mechanical aortic valve replacement performed with concomitant coronary artery bypass grafting. Ann Thorac Surg. 2002;74:1098-106.

88. Messika-Zeitoun D, Yiu SF, Grigioni F, Enriquez-Sarano M. Role of echocardiography in the detection and prognosis of ischemic mitral regurgitation. Rev Esp Cardiol. 2003;56:529-34.

89. Aklog L, Filsonfi F, Flores KQ, Chen RH, Cohn LH, Nathan NS, et al. Does coronary artery bypass grafting alone correct moderate ischemic mitral regurgitation? Circulation 2001;104 Suppl I:I6875 .

90. Jouan J, Tapia M, Cook RL, Lausec E, Acar C. Ischemic mitral valve prolapse: mechanisms and implications for valve repair. Eur J Cardiothorac Surg. 2004;26:1112-7.

91. Bax JJ, Braun J, Somer ST, Klautz R, Holman ER, Versteegh MIM, et al. Restrictive annuloplasty and coronary revascularization in ischemic mitral regurgitation results in reverse left ventricular remodelling. Circulation. 2004;110 Suppl II:II103-8.

92. Menicanti L, Di Donato M. The Dor procedure: what has changed after fifteen years of clinical procedure? J Thorac Cardiovasc Surg. 2002;124:886-90

93. Menicanti L, Di Donato M. Surgical left ventricle reconstruction: pathophysiologic insights, results and expectation from the STICH trial. Eur J Cardiothorac Surg. 2004;26 Suppl:S43-7. 